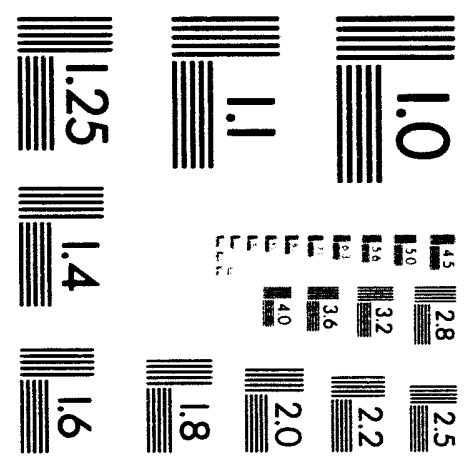



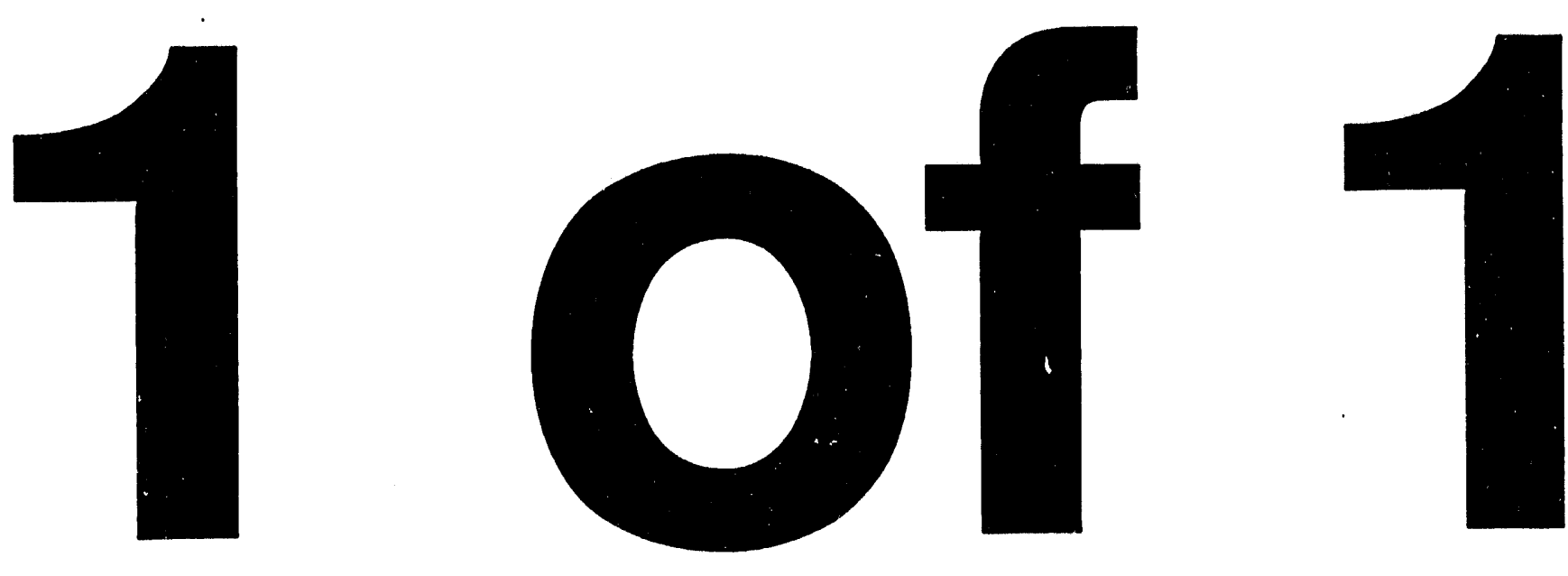

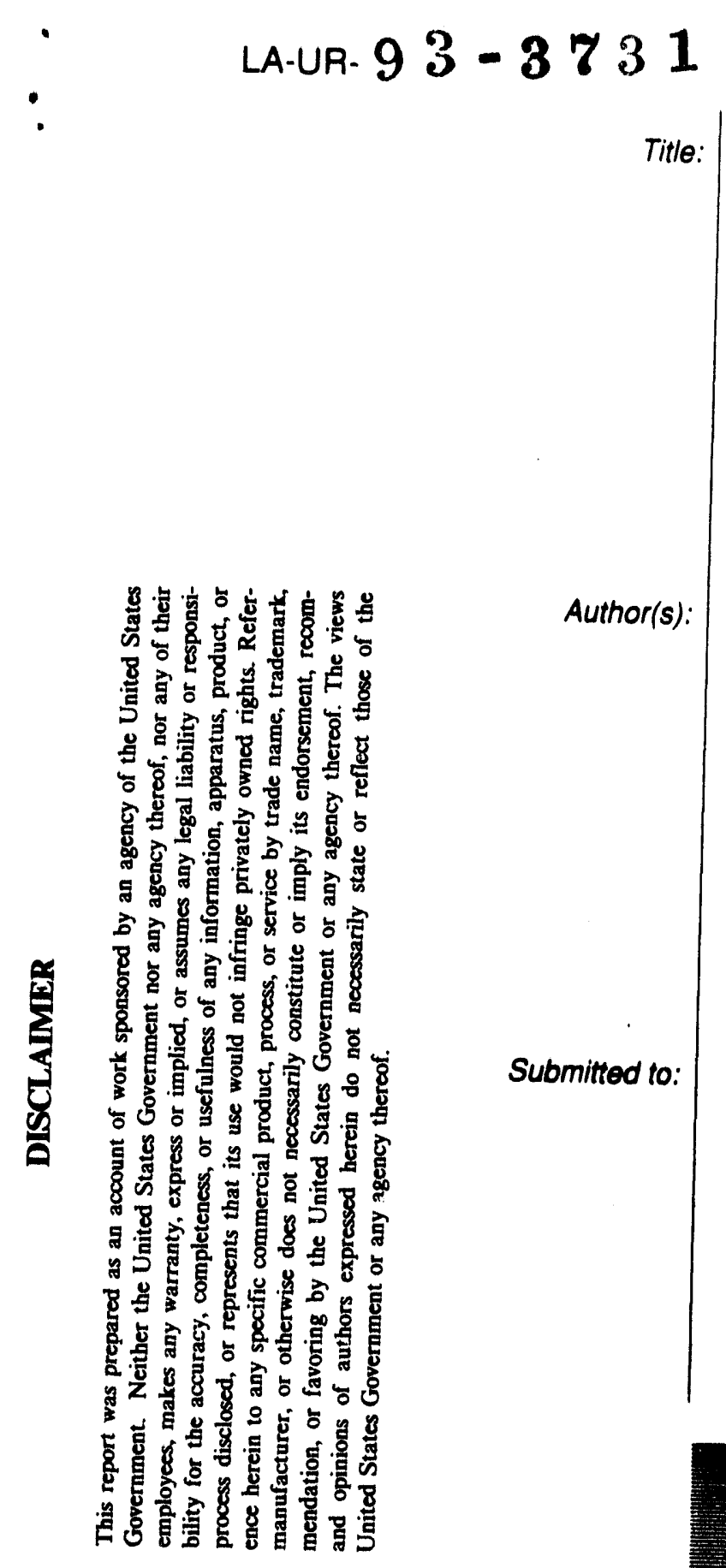

GENERALIZED SQUEEZED STATES FROM GENERALIZED COHERENT STATES

MICHAEL MARTIN NIETO, T-8, MS B285

LOS ALAMOS NATIONAL LABORATORY

LOS ALAMOS, NM 87545

PROCEEDINGS OF THE INTERNATIONAL SYMPOSIUM

ON COHERENT STATES HELD AT OAK RIDGE NATIONAL

LABORATORY, OAK RIDGE, TN, JUNE 14-17, 1993.

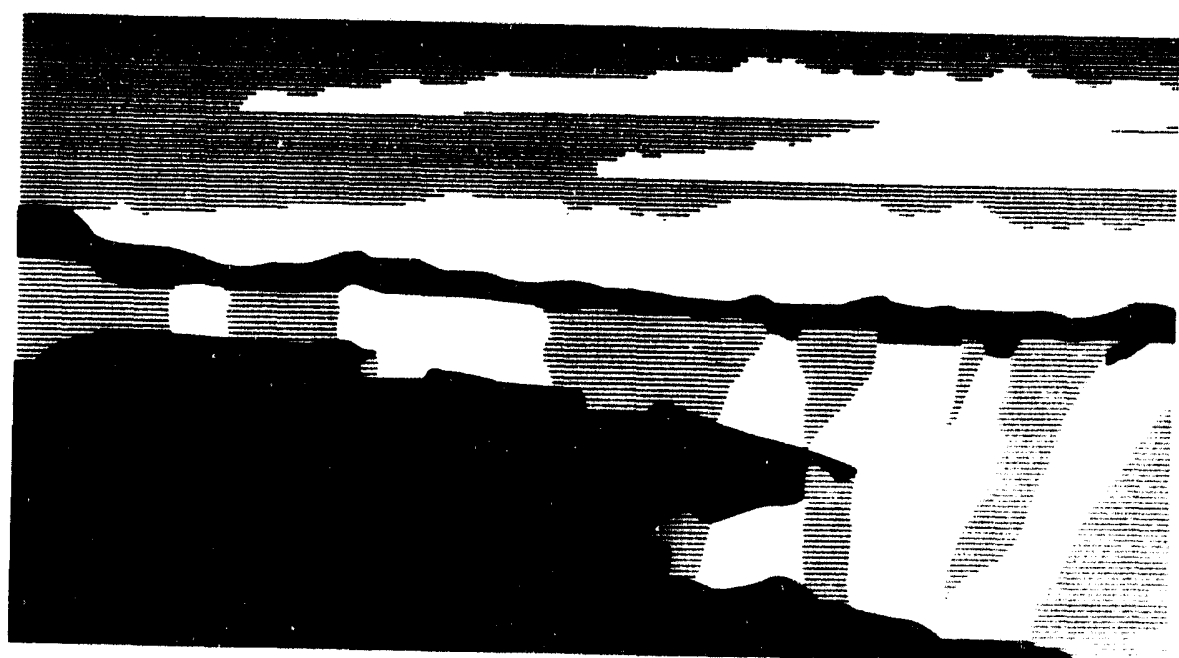

Los Alamos National Laboratory, an aftirmative actionequal opportunity employer, is operated by the Univeraity of Calltornia for the U.S. Department of Energy publish or reproducs the published form of this of this article, the publiaher recognizes that the U.S. Government retains a nonexclusive, poyalty-free license to requests that the pusliaher identhy this anticts se work pertormed under the ausedo so, for U.S. Governmem purposes. The Los Alamos National Laboratory 


\title{
GENERALIZED SQUEEZED STATES FROM GENERALIZED COHERENT STATES
}

\author{
Michael Martin Nieto ${ }^{1}$ \\ Theoretical Division, Los Alamos National Laboratory \\ University of California \\ Los Alamos, New Mexico 87545, U.S.A.
}

\begin{abstract}
Both the coherent states and also the squeezed states of the harmonic oscillator have long been understood from the three classical points of view: the 1) displacement operator, 2) annihilation- (or ladder-) operator, and minimum-uncertainty methods. For general systems, there is the same understanding except for ladder-operator and displacement-operator squeezed states. After reviewing the known concepts, I propose a method for obtaining generalized minimum-uncertainty squeezed states, give examples, and relate it to known concepts. I comment on the remaining concept, that of general displacement-operator squeezed states.
\end{abstract}

\section{Introduction}

As we all know, Glauber, Klauder, and Sudarshan produced the modern era's seminal works on coherent states of the harmonic oscillator $[1,2,3,4]$. There came to be three classical definitions of these coherent states, from 1) the displacement-operator method, 2) the annihilation-operator method which I will relabel the ladder-operator method, and 3) the minimum-uncertainty method. Today coherent states are important in many fields of theoretical and experimental physics [5, 6].

Generalizations of these states appeared in two areas. One was to "two-photon states" [i] , states which were rediscovered under many names. In 1979 they were first called "squeezed states" [8]. In recent times these states have become of more and more interest $[9,10]$. This is especially true in the fields of quantum optics [11] and gravitational wave detection [12]. The squeezed states of the harmonic oscillator

\footnotetext{
'Email: mmn@̄pion.lanl.gov
} 
can also be equivalently defined by appropriate generalizations of the three classical definitions of the coherent states.

The oti.ts generalization was to non-harmonic oscillator systems. From the group theory or operator point of view. generalized coherent states for Lie groups were widely studied from the displacement operator and annihilation operator methods [3. 13].

In 1978 I. along with Mike Simmons and Vincent Gutschick, began a program to find the coherent states for arbitrary potentials from a minimum-uncertainty point of view. You give us the potential, be it Morse, Pöschl-Teller, Coulomb, or whatever. and we'll give you the coherent states. What came as a byproduct was the realization that along the way we had also found the appropriate generalization to the squeezed state. Further, we found that for arbitrary systems the three methods no longer necessarily gave the same coherent and/or squeezed states.

This all is in the past, from the theme of this Symposium. It leaves appropriate generalizations of squeezed states from the ladder-operator and displacement-operator points of view to be found.

The present deals with work which Rod Truax and I have recently reported on [14]. (Indeed, in this Proceedings I draw upon much of the contents of Ref. [14], especially in Section 3.) We proposed a generalization of squeezed states using the ladder-operator method. This method was also connected to the minimumuncertainty method and some aspects of special-case displacement-operator squeezed states which have been obtained. It was also found that, as expected, the generalized squeezed states from the different methods could be equivalent, but need not be. (I note that connections of these ideas to Rydberg wave packets and other quantum systems have been made elsewhere $[15,16]$.)

This leaves the future. What remains to be done is to find generalized squeezed states from the displacement-operator method.

\section{Past}

\subsection{Coherent states for the harmonic oscillator}

I begin by reviewing the coherent states for the harmonic oscillator. As is well-known. there are three standard definitions of these states, which are equivalent.

1) Displacement-Operator Method. The coherent states can be obtained by applying the unitary displacement operator on the ground state $[3,2]$ :

$$
|\alpha\rangle \equiv D(\alpha)|0\rangle=\exp \left[\alpha a^{\dagger}-\alpha^{*} a\right]|0\rangle=\exp \left[-\frac{1}{2}|\alpha|^{2}\right] \sum_{n} \frac{\alpha^{n}}{\sqrt{n !}}|n\rangle
$$

where $|n\rangle$ are the number states. 
2) Ladder- (or Annihilation-) Operator Method. The coherent states can also be defined as the eigenstates of the destruction operator:

$$
a|\alpha\rangle=\alpha|\alpha\rangle
$$

This follows from Eq. (1), since

$$
0=D(\alpha) a|0\rangle=(a-\alpha) D(\alpha)|0\rangle=(a-\alpha)|\alpha\rangle .
$$

These states are the same as the displacement-operator coherent states.

3) Minimum-Uncertainty Method. This method harks back to Schrödinger's discovery of the coherent states [1i]. Recall that Schrödinger wanted to find states which maintained their shapes and followed the classical motion. For the harmonic oscillator, these are the states which minimize the uncertainty relation

$$
[x, p]=i \hbar, \quad(\Delta x)^{2}(\Delta p)^{2} \geq \frac{1}{4} \hbar^{2}
$$

subject to the constraint that the ground state is a member of the set. In wave function language $(\hbar=\omega=m=1)$ they are described by

$$
\psi_{c s}(x)=[\pi]^{-1 / 4} \exp \left[-\frac{\left(x-x_{0}\right)^{2}}{2}+i p_{0} x\right]
$$

That the states of Eqs. (1) and (5) are the same can be demonstraled by using the generating function for the Hermite polynomials along with the identifications

$$
\sqrt{2} \Re(\alpha)=x_{0}, \quad \sqrt{2} \Im(\alpha)=p_{0} .
$$

Observe that of the four original parameters, $\langle x\rangle,\left\langle x^{2}\right\rangle,\langle p\rangle$, and $\left\langle p^{2}\right\rangle$. only two remain. $\Re(\alpha)$ and $\Im(\alpha)$. That is firstly because the inequality in the uncertainty relation has been satisfied. The remaining three parameter set of states is restricted to two parameters by demanding that the ground state (which corresponds to zero motion) must be a member of the set.

\subsection{Squeezed states of the harmonic oscillator}

The above three methods also yield equivalent squeezed states for the harmonic oscillator.

1) Displacement-Operator Method. In this method one applies the "squeeze" or $\mathrm{SU}(1,1)$ displacement operator on the coherent state:

$$
D(\alpha) S(z)|0\rangle=|(\alpha, z)\rangle, \quad S(z)=\exp \left[z K_{+}-z^{*} K_{-}^{*}\right],
$$


where $K_{+}, K_{-}$, and $K_{0}$ form an su(1,1) algebra amongst themselves:

$$
\begin{gathered}
K_{+}=\frac{1}{2} a^{\dagger} a^{\dagger}, \quad K_{-}=\frac{1}{2} a a, \quad K_{0}=\frac{1}{2}\left(a^{\dagger} a+\frac{1}{2}\right), \\
{\left[K_{0}, K_{ \pm}\right]= \pm K_{ \pm}, \quad\left[K_{+}, K_{-}\right]=-2 K_{0} .}
\end{gathered}
$$

The ordering of $D S$ vs. $S D$ in Eq. $(\vec{l})$ is unitarily equivalent, amounting to a change of parameters:

$$
D(\alpha) S(z)=S(z) D(\vartheta), \quad \gamma=\alpha \cosh r-\alpha^{*} e^{i \theta} \sinh r
$$

where $z=r e^{i \theta}$.

2) Ladder-Operator Method. For the harmonic oscillator, this method again follows from the displacement-operator method. Combining the Bogoliubov transformation [18],

$$
S^{-1} a S=(\cosh r) a+e^{i \theta}(\sinh r) a^{\dagger}
$$

with Eq. (10). one has that

$$
\left[(\cosh r) a-e^{i \theta}(\sinh r) a^{\dagger}\right]|(\alpha, z)\rangle=\gamma|(\alpha, z)\rangle
$$

One now sees why I relabel this method the ladder-operator method. For the squeezed states one needs both the raising (creation) and lowering (annihilation) operators.

3) Minimum-Uncertainty Method. From this point of view, the transition from coherent to squeezed states is intuitively simple. These states minimize the $x-p$ uncertainty relation, without the added restriction that the ground state (Gaussian) is a member of the set. That is, these are a three parameter set of states, which are the Gaussians of all widths:

$$
\psi_{s s}(x)=\left[\pi s^{2}\right]^{-1 / 4} \exp \left[-\frac{\left(x-x_{0}\right)^{2}}{2 s^{2}}+i p_{0} x\right]
$$

These squeezed states are equivalent to those obtained from the other formulations. This can be verified by combining Eqs. (12) and (13) with the relationships

$$
x=\frac{\left(a+a^{\dagger}\right)}{\sqrt{2}}, \quad p=\frac{\left(a-a^{\dagger}\right)}{i \sqrt{2}} .
$$

The remaining relationships among the parameters are

$$
z=r e^{i \theta}, \quad r=\ln s .
$$

(The phase, $\theta$, is an initial time-displacement.) 


\subsection{Generalized (displacement- and ladder-operator) coherent states}

When one considers coherent states for general systems one finds that the coherent states from all three methods are not, in general. equivalent. although they may be in particular cases.

1) Displacement-Operator $M$, ind. The generalization of this method to arbitrary Lie groups has a long history $[5,6,3,13]$. (Supersymmetric extensions of it also exist [19].) One simply applies the displacement operator, which is the unitary exponentiation of the factor algebra. on to an extremal state. That is, let $T$ be a unitary irreducible representation of the group $G$ on a Hilbert space and let $\left|\psi_{0}^{\prime}\right\rangle$ be a fixed vector in the space. Let $G_{0}$ be the stability group; i.e.,

$$
T\left(G_{0}\right)\left|\psi_{0}^{\prime}\right\rangle=e^{i \alpha\left(G_{0}\right)}\left|\psi_{0}\right\rangle .
$$

Then

$$
\left|\psi_{g}\right\rangle=T\left(G / G_{0}\right)\left|\psi_{0}\right\rangle
$$

are the coherent states.

2) Ladder-Operator Method. The generalization to arbitrary Lie groups is straight forward, and has also been widely studied [5.6]. One obtains the eigenstates of the generalized lowering-operator (assuming there is an extremal state below):

$$
A_{-}|\delta\rangle=\delta|\delta\rangle \text {. }
$$

\subsection{Generalized (minimum-uncertainty) coherent and squeezed states}

In the program I described in the introduction, we wanted to find the coherent states for arbitrary potential systems. We were motivated by the physics of Schródinger. We wanted to find states which follow the classical motion as well as possible and which maintain their shapes as well as possible. We felt that meant there was an associated uncertainty relation. but in which variables? Our answer was found in a manner completely backwards from the way I now present it, but such is often the case with physics. In fact, along the way, this method also yields the squeezed states for genera! potential systems $[20,21]$.

One starts with the classical Hamiltonian problem and transforms it into the "natural classical variables," $X_{c}$ and $P_{c}$, which vary as the sin and the cos of the classical $\omega t$. The Hamiltonian is therefore of the form $P_{c}^{2}+X_{c}^{-2}$. One then takes these natural classical variables and transforms them into "natural quantum operators." Since these are quantum operators, they have a commutation relation and uncertainty relation:

$$
[X, P]=i G, \quad(\Delta X)^{2}(\Delta P)^{2} \geq \frac{1}{4}\langle G\rangle^{2}
$$


The states that minimize this moncertainty relation are given by the solutions to the equation

$$
Y_{\psi_{s s}} \equiv\left(X+\frac{i\langle G\rangle}{2(\Delta P)^{2}} P\right) \psi_{s s s}=\left(\left\langle\mathrm{H}^{\cdot}\right\rangle+\frac{i\langle G\rangle}{2(\Delta P)^{2}}\langle P\rangle\right) \psi_{s s s}
$$

Note that of the four parameters $\left\langle\mathrm{I}^{*}\right\rangle,\langle P\rangle,\left\langle P^{2}\right\rangle$, and $\langle G\rangle$, only three are independent because they satisfy the equality in the uncertainty relation. Therefore.

$$
(\mathrm{I}+i B P) \psi_{s s}=C \psi_{s s}, \quad B=\frac{\Delta \mathrm{X}^{*}}{\Delta P}, \quad C=\left\langle\mathrm{X}^{-}\right\rangle+i B\langle P\rangle .
$$

Here $B$ is real and $C$ is complex. We called these states, $\psi^{\prime s s}(B, C)$, the minimumuncertainty states for general potentials $[20,21]$. However, using the parlance accepted later. they are the squeezed states for general potentials [10]. Then $B$ can be adjusted to $B_{0}$ so that the ground eigenstate of the potential is a member of the set. Then these restricted states. $\psi_{s s}\left(B=B_{0}, C\right)=\psi_{c s}\left(B_{0}, C\right)$, are the minimumuncertainty coherent states for general potentials.

It can be intuitively understood that $\psi_{s s}(B, C)$ and $\psi_{s s}\left(B_{0}, C\right)$ are the squeezed and coherent states by recalling the situation for the harmonic oscillator. The coherent states are the displaced ground state. The squeezed states are Gaussians that have widths different than that of the ground state Gaussian, which are then displaced.

\section{Present: Generalized Ladder-Operator Squeezed States}

As we have discussed, general annihilation-operator (or ladder-opirator) coherent states are the eigenstates of the lowering operator (given a lowest extremal state). We now propose a generalization to squeezed states, including those for arbitrary symmetry systems: The general ladder-operator squeezed states are the eigenstates of a linear combination of the lowering and raising operators.

I note that the success of this method will not be totally surprising. In many exactly solvable potential systems, the natural quantum operators of the minimumuncertainty method were found to be Hermitian combinations of the $\mathrm{n}$-dependent raising and lowering operators [20,21]. Here, however, one must generalize to full operators: $n \rightarrow n(H)$. Furthermore, in other harmonic-oscillator-like systems, with a Bogoliubov transformation, this method applies. (See below.)

I will proceed by showing how the minimum-uncertainty method for obtaining generalized squeezed states can be used as an intuitive tool to aid in understanding the ladder-operator method for obtaining generalized squeezed states. I will do this

with two specific examples. Once that is done, the ladder operator method can 
be applied to general symmetry systems, independent of whether they come from a Hamiltonian system in the manner of the minimum-uncertainty method above. Such is our third example.

\subsection{Example I: The harmonic oscillator.}

First let us re-examine the harmonic oscillator, starting from the minimum-uncertainty method. Here $X$ and $P$ are obviously $x$ and $p$. Then we have

$$
Y=x+s^{2} \frac{d}{d x}
$$

where we have presciently labeled $B$ as $s^{2}$. (For the limit to coherent states, it turns out that $B=1$.)

Now writing $x$ and $p$ in terms of creation and annihilation operators, we find

$$
\left.\sqrt{2}\left[a\left(\frac{1+s^{2}}{2}\right)+a^{\dagger}\left(\frac{1-s^{2}}{2}\right)\right] \psi s s\left(s^{2}, x_{0}+i s^{2} p_{0}\right)=\left[x_{0}+i s^{2} p_{0}\right] \psi^{\prime} s s^{2}, s_{0}+i s^{2} p_{0}\right)
$$

Therefore, the squeezed states are eigenstates of a linear combination of the annihilation and creation operators. Specifically, these states are as given in Eq. (13).

$$
\psi^{\prime} \cdot s(x)=\left[\pi s^{2}\right]^{-1 / 4} \exp \left[-\frac{\left(x-x_{0}\right)^{2}}{2 s^{2}}+i p_{0} x\right]
$$

\subsection{Example II: The harmonic oscillator with centripetal barrier.}

I now discuss the symmetry of the harmonic oscillator with centripetal barrier. Previously, the coherent states for this particular example were found with the minimumuncertainty method, but not the squeezed states [21]. Therefore, it is a useful system since, at the end, we can relate our results to the coherent states obtained from the minimum-uncertainty method.

This system contains an su(1,1) algebra [22]. Its elements are

$$
L_{ \pm}=\frac{1}{4 \nu} \frac{d^{2}}{d z^{2}} \mp \frac{1}{2} z \frac{d}{d z} \mp \frac{1}{4}+\frac{\nu}{4} z^{2}-\frac{\nu}{4 z^{2}}
$$




$$
L_{11}=\frac{H}{41^{\prime}}+\frac{11}{2} . \quad H=-\frac{11^{2}}{1:^{2}}+1^{2}\left(\frac{1}{2}-:\right)^{2}
$$

[n terms of the $X$ ind $P$ minimum-mucerainty operators [21]. We find

$$
X=\frac{L_{-}+L_{+}}{\nu}=:^{2}-\left(1+\frac{H}{2 \nu^{2}}\right) . \quad P=\frac{2\left(L_{-}-L_{+}\right)}{i}=\frac{1}{i}\left[2 z \frac{1}{d_{i}}+1\right] .
$$

Therefore. the spleeved states for this system are formed by the solution to the equation

$$
0=\left[\eta \frac{r^{2}}{d ! \eta^{2}}+\left(\frac{1}{2}+2, B ! \eta\right) \frac{d}{d !}+\frac{1}{4}\left(\eta-\frac{\nu^{2}}{\eta}+2 B \nu\right)-\frac{\nu C}{2}\right] \| .
$$

where we have changed rariables to $y=\nu:^{2}$. The squeezed state solutions to this reflation are

$$
\therefore=. \lambda+x)[-y(\nu B+-1)]\left[y^{\lambda+\frac{1}{3}}\right] \Phi\left(\left[\frac{\nu C}{4 \gamma}+\frac{1}{2}\left(\lambda+\frac{3}{2}\right)\right] .\left[\lambda+\frac{3}{2}\right] ; 2 \gamma y\right) .
$$

Whore $\Phi(a, b ; c)$ is the confluent hypergeometric function $\sum_{n=0}^{x} \frac{(a)_{n} r^{n}}{(b)_{n} n !} \cdot \hat{i}=\sqrt{\nu^{2} B^{2}-\frac{1}{i}}$.

and $\lambda(\lambda+1)=\nu^{2}$. In the limit where $B \rightarrow 1 /(2 \nu)$, these become the colierent states give'n in Ref. [21].

$$
\therefore \therefore=\left[\frac{2 \nu^{1 / 2} e^{-\nu \lambda\left(C^{\prime}\right)}}{I_{\lambda+1 / 2}(\nu|C|)}\right]^{1 / 2} e^{-y / 2} y^{1 / 4} I_{\lambda+1 / 2}\left(\left(2 \nu C^{\prime} ! y\right)^{1 / 2}\right)
$$

where $I$ is the modified Bessel function.

\subsection{Example III: The squeeze algebra.}

We now consider a symmetry system which does not have as its origin a Hamiltonian sistem. Wi cousider the su(1.1) symmetry of Eqs. (8. 9). Our ladder-operator squeezed states are thus the solutions to

$$
\left[\left(\frac{1+s}{2}\right) a a+\left(\frac{1-s}{2}\right) a^{\dagger} a^{\dagger}\right] \psi_{s .4}=3^{2} \iota_{s .4 .}
$$




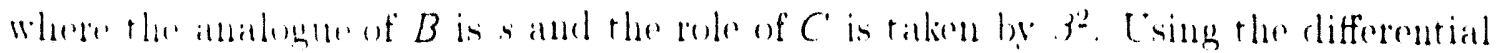
representations of the harklere operators. Eq. (31) an be writen in the form

$$
\left[\frac{d^{2}}{d y^{2}}+2 y s \frac{d}{d ! y}+y^{2}+\left(s-2.3^{2}\right)\right] 4 x=0 .
$$

() berve that the ladder operators rase and lower the mumber states by two units. Therefore, there will be two solutions to this equation. one containing only even number states and one rontaining only odd number states. We will designate these us l'ess and l'o.s. These solutions are

$$
\begin{aligned}
& U_{E s s}=V_{E} \exp \left[-\frac{-y^{2}}{2}\left(s+\sqrt{s^{2}-1}\right)\right] \Phi\left(\left[\frac{1}{4}+\frac{3^{2}}{2 \sqrt{s^{2}-1}}\right] \cdot \frac{1}{2} ; y^{2} \sqrt{s^{2}-1}\right) . \\
& \left.U_{0 . s}=V_{0} y \exp \right)\left[-\frac{-y^{2}}{2}\left(s+\sqrt{s^{2}-1}\right)\right] \Phi\left(\left[\frac{3}{4}+\frac{3^{2}}{2 \sqrt{s^{2}-1}}\right] \cdot \frac{3}{2}: y^{2} \sqrt{s^{2}-1}\right) .
\end{aligned}
$$

In the limit $s \rightarrow 1$. Phese berome the even and odd coherent states:

$$
\begin{aligned}
& c^{\prime} E_{\mathrm{r} \cdot s}=\left[\frac{e^{-3^{3}}}{\pi^{1 / 2} \cosh |, 3|^{2}}\right]^{1 / 2} \exp \left[-\frac{1}{2} y^{2}\right] \cosh (\sqrt{2} 3 y) \\
& r^{\prime}(0) s=\left[\frac{e^{-y^{2}}}{\pi^{1 / 2} \sinh |;|^{2}}\right]^{1 / 2} \exp \left[-\frac{1}{2} y^{2}\right] \sinh (\sqrt{2} 3 y)
\end{aligned}
$$

lsing genrating formulae, these can be written in the number-state basis as

$$
\begin{aligned}
& \left.{ }_{4} \text { Ecs }=|\cosh |,\left.3\right|^{2}\right]^{-1 / 2} \sum_{n=0}^{\infty} \frac{3^{2 n}}{\sqrt{(2 n) !}}|2 n\rangle . \\
& u_{0} 0_{c s}=\left[\sinh |;|^{2}\right]^{-1 / 2} \sum_{n=0}^{\infty} \frac{3^{2 n+1}}{\sqrt{(2 n+1) !}}|2 n+1\rangle \text {. }
\end{aligned}
$$

Cp to the normalization, these are the "even and odd coherent states" previously found in Ref. [23]. Although this system did not come from a Hamiltonian, one could 


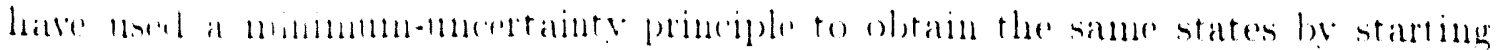

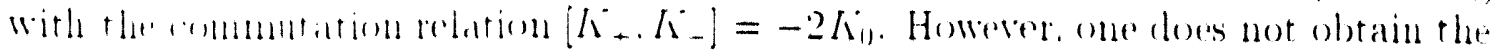

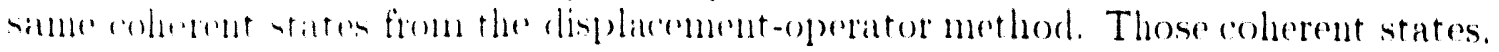

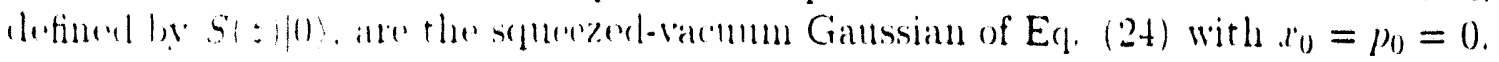

The above three examples have all been cases where $t_{-}=\left(. t_{+}\right)^{\dagger}$. Sometimes that is not the rase, at in rentain potential systems whose eigenenergies are not equally spaced [20. 21]. Then. as in E(1. (26), one should use the operator form for "1": $A_{n} \rightarrow A_{n(H)}$. to comnect to the minimum-nncertainty method. In these cases. the ladder-operator coherent and squeezed states can be different than. though rolated to. their minimum-mucertainty counterparts.

\section{Future: Generalized Displacement-Operator Squeezed States?}

Although the displanement-operator method is the natural one for defining coherent states of Lie gromps. there is as yet no well-known general extension of this method to define general displacement-operator squeezed states. It has basically only been applied to harmonic-oscillator-like systems [0.10].

This has been touched upon in discussions [24] about higher-order generalizations of the "squeeze operator." $S(:)$. In particular, although harmonic-oscillatorlike systems admit squeeze operators (or Bogoliubov transformations) connecting the displacement-operator and ladder-operator methods [25. 26], the appropriate generalization of these squeeze operators have not been found. Therefore, for now, the ladderoperator method is generally connected only to the minimum-nncertainty method.

Cote also that for finite-dimensional representations, such as for angular momentum colnerent states. the ladder-operator method does not allow a solution for coherent states. although the displacement-operator method does [25]. Contrariwise, for scpueezed states. we observe that the opposite is true.

It thus remains for the future to find generalized squeezed states from the displacement operator method. I have been given some helpful advice by a number of people at this Simposium, including John Klander and Arthur Wightman. but the solution remains to be found. Hopefully, at the next Symposium something further (aul be said. 


\section{References}

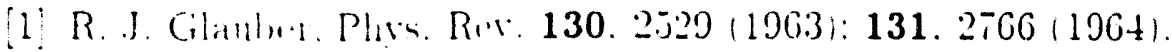

[2] J. R. Klauder. J. Math. Phys 4. 10.5.5 (1063): ibid. 5. 17 (1964).

[3] J. R. Klatuder. J. Math. Phes 4. 1058 (1063).

[H] E. C. G. Sularshan. Physs. Rer. Lett. 10. 227 (1063).

[j] J. R. Klander and B.-S. Skagerstam. Coherent States - Applications in Physics and Mathematical Physics (World Scientific. Singapore. 1085):

[6] II.-.M. Zhang. D. H. Feng. and R. Gilmore. Rev. Mod. Phys. 62. 86 T (1990).

ii] P. P. Yuen. Phỵs. Rev. A 13. 2226 (1976).

[8] J. N. Hollenhorst. Physs. Rer. D 19. 1669 (1079):

[9] D. Han. Y. S. Kim. and W: W: Zachary. eds.. Workshop on Squeezed States and Uncerfuinty Relations. N.AS.A Conference Publication 3135 (...AS.A. Washington. D. C.. 1992).

[10] For a pedagogical review. spe .I. .I. Nieto. in: Frontiers of Nonequilibrium Statistical Physics. eds. G. T. Moore and .I. O. Scully (Plenum. New York. 1986) p. $28 \%$.

[11] Workshop on Squeezed States and Uncertainty Relations. ed. by D. Han. Y. S. Kim. and W. WV. Zachary. N.AS.A Conference Publication 3135 (1992).

[12] C. M. Cares. K. S. Thorne. R. II. P. Drever. I. D. Sandberg. and M. Zimmerman. Rev. .Iod. Physs. 52. 341 (1980).

[13] A. O. Barut and L. Girardello. Commun. Math. Phys. 21. 4 (1071): A. M. Perelomor. Commun. Math. Physs. 26. 222 (1972): Generalized Coherent States and Their Applications (Springer-Verlag. Berlin. 1986): R. Gilmore. Lie Groups. Lit. Algebras. and Some of Their Applications (Wiley. New York. 19it).

[14] M. M. Nieto and D. R. Truax. Phys. Rev. Lett. (submitted).

[1.j] M. M. Yieto. Quantum Optics (in press).

[16] R. Bluhm and $\mathrm{V}$. A. Kosteleckí. Indiana Lniversity preprints ILHET-255 and 256 .

[17] E. Schrödlinger. Naturwiss. 14. 66+ (1926).

[18] ‥ Bogolubor. J. Physs. [SSR 11. $292(194 i)$. 


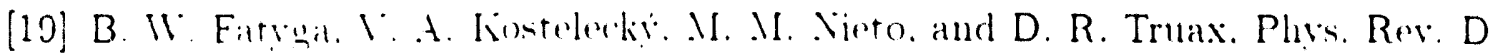

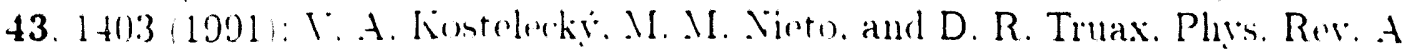
(10) finlilinhed).

[20] M. .I. Xieto and L. M. Simmons. Jr.. Plỵs. Rev. Lett. 41. 207 (1978): Phys, Rer. D 20. 1321 (1970). the first of a series concluding with . . . Nieto. L. M. Simmons, Ir.. and V. P. Gutschick. Plivs. Rev. D 23. 927 (1981): M. M. Nieto. in Ref. [.5]. 1). +29. gives a summary of this prom. :

[21] .I. .I. Nieto and L. .1. Simmons. Jr.. Phys. . D 20, 1332 (1979).

[22] D. R. Truax. J. Math. Phỵs. 22 1050 (1981).

[23] I'. I’. Dodonor. I. A. Malkin, and I’. I. Man ko. Physica 72. 59 T (1974).

[24] R. A. Fisher. M. .I. Nieto. and I'. D. Sand'serg. Phys. Rev. D 29, 1107 (1984): G. D Ariano and .I. Rasetti. Physs. Rev. D 35. 1239 (108T): J. Ratriel. M. Rasetti. and A. I. Soloman. Phịs. Rer. D 35. 1248 (198i) and references therein.

[25] T. S. Santhanam. in: Einstein Centennial Week Symposium on Symmetries in Science : ed. by. B. Gruber and R. S. Millman (Plenum. NY. 1980). p. 33T.

[26] G. S. Agarwal and R. R. Puri. Phys. Rev. A 41. 3782 (1990): A. T. Filippov. D. Gangopadhyay, and A. P. Isaev. J. Phys. A 24. L63 (1991). 

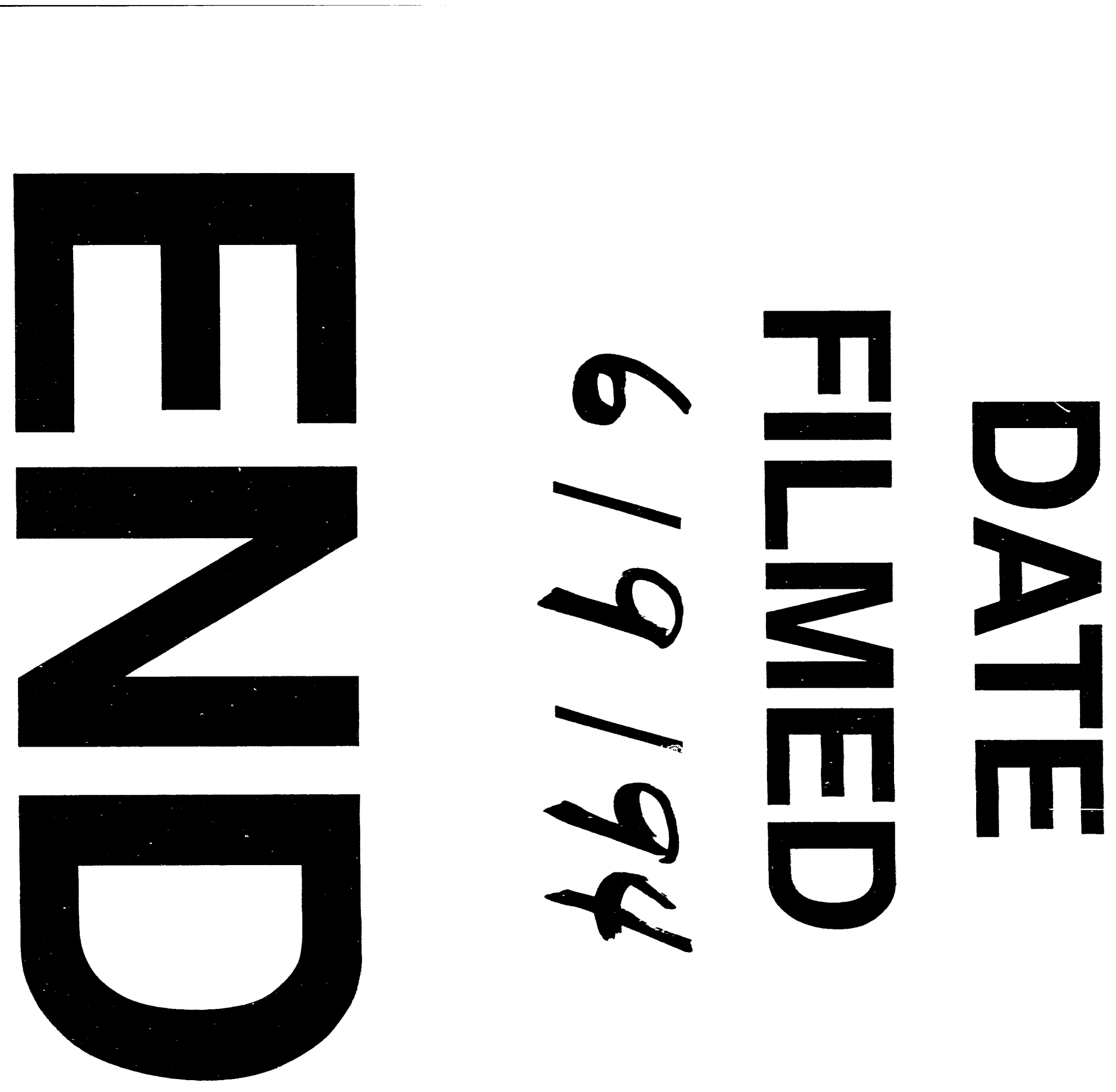

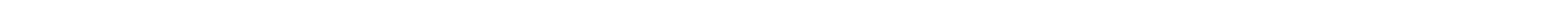